\title{
Vesicle electrohydrodynamic simulations by coupling immersed boundary and immersed interface method
}

\author{
Wei-Fan Hu* Ming-Chih Laił Yunchang Seolł Yuan-Nan Young $\$$
}

\begin{abstract}
In this paper, we develop a coupled immersed boundary (IB) and immersed interface method (IIM) to simulate the electrodeformation and electrohydrodynamics of a vesicle in Navier-Stokes leaky dielectric fluids under a DC electric field. The vesicle membrane is modeled as an inextensible elastic interface with an electric capacitance and an electric conductance. Within the leaky dielectric framework and the piecewise constant electric properties in each fluid, the electric stress can be treated as an interfacial force so that both the membrane electric and mechanical forces can be formulated in a unified immersed boundary method. The electric potential and transmembrane potential are solved simultaneously via an efficient immersed interface method. The fluid variables in Navier-Stokes equations are solved using a projection method on a staggered MAC grid while the electric potential is solved at the cell center. A series of numerical tests have been carefully conducted to illustrate the accuracy and applicability of the present method to simulate vesicle electrohydrodynamics. In particular, we investigate the prolate-oblate-prolate (POP) transition and the effect of electric field and shear flow on vesicle electrohydrodynamics. Our numerical results are in good agreement with those obtained in previous work using different numerical algorithms.
\end{abstract}

Keywords: Immersed boundary method; immersed interface method; vesicle electrohydrodynamics; leaky dielectric model; Navier-Stokes equations

\section{Introduction}

Vesicle electrohydrodynamics (dynamics of the fluid flow around a vesicle under an external electric field) has received much attention due to its wide applications in both biological and biomedical systems that involve vesicle manipulations, such as electroporation [16, 34], creating crack [18], and electrofusion [13]. A comprehensive review for more applications can be found in $[3,4]$. In this paper, our aim is to develop a numerical scheme for simulations

\footnotetext{
${ }^{*}$ Corresponding author, Department of Applied Mathematics, National Chung Hsing University, 145, Xingda Road, Taichung City 402, Taiwan, wfhu@nchu.edu.tw

${ }^{\dagger}$ Center of Mathematical Modeling and Scientific Computing \& Department of Applied Mathematics, National Chiao Tung University, 1001, Ta Hsueh Road, Hsinchu 300, Taiwan, mclai@math.nctu.edu.tw

${ }^{\ddagger}$ National Center for Theoretical Sciences, No. 1, Sec. 4, Road. Roosevelt, National Taiwan University, Taipei 10617, Taiwan, ycseol@ntu.edu.tw

${ }^{\S}$ Department of Mathematical Sciences, New Jersey Institute of Technology, Newark, NJ 07102, USA, yyoung@njit.edu
}

(C) 2016. This manuscript version is made available under the Elsevier user license http://www.elsevier.com/open-access/userlicense/1.0/ 
of vesicle electrodeformation; in particular, we focus on a leaky dielectric vesicle suspended in another leaky dielectric fluid under a uniform DC (direct current) electric field.

Various theoretical models have been developed for the electrohydrodynamics and electrodeformation of a non-porated vesicle under an external electric field. For a DC electric field, theoretical models have been developed for a slightly deformed vesicle $[10,11,16,37]$ and a spheroidal vesicle $[35,46,47]$. The extension to a vesicle under an AC (alternative current) electric field can be found in $[6,12,32,43]$. These theoretical results are limited by the model assumptions such as small deformation from a nearly spherical vesicle and the vesicle can only take a spheroidal shape.

In experiments the dependence of the vesicle shape and dynamics on the physical parameters provides a means to measure the membrane bending modulus, membrane electrical capacitance, and membrane conductance $[2,3,4,39]$. For example, when the vesicle interior is less conductive than the exterior fluid, the vesicle might undergo a prolate-oblate-prolate (POP) transition [39].

Numerical methods for simulating vesicle electrohydrodynamics are far and few between. Focusing on Stokes flow dynamics, McConnell and Miksis developed a boundary-integral numerical scheme for simulating the two-dimensional vesicle electrohydrodynamics [30]. They further extended their work to study the vesicle breathing dynamics under an AC electric field [31]. Using a level-set/immersed interface hybrid approach, Kolahdouz and Salac proposed a numerical scheme for three-dimensional vesicle electrohydrodynamics in Navier-Stokes flow [22] and used it to study the effects of different fluid and membrane properties on the POP transition [23]. The immersed interface method is used to solve the electric potential [21] and the level set method is used to solve for the fluid flow [22]. In the present work, our hybrid numerical scheme uses an immersed interface method for the electric potential and an immersed boundary method for the Navier-Stokes flow.

The new numerical implementations and techniques reported here for both the immersed interface method (for the electric part) and the coupling between electric and fluid flow via immersed boundary method are quite different from the schemes in [22]. Firstly, based on our previous work for simulating drop electrohydrodynamics [15], we cast the Maxwell stress tensor as an electric interfacial force (the jump of Maxwell tensor across the interface) rather than a volume force in the equations so that the vesicle membrane force and interfacial electric stress can be formulated in a unified immersed boundary framework. This step is significantly advantageous because it allows us to compute the electric force in an accurate manner, and also greatly simplifies the numerical algorithm for the full system. Secondly, we develop an augmented immersed interface method for solving the electric potential. Different from drop electrohydrodynamics, the elliptic interface equation for the electric potential for the vesicle electrohydrodynamic couples a time-varying transmembrane potential. As a result, the vesicle transmembrane potential is solved simultaneously with the electric potential.

Thirdly, we treat the vesicle membrane as a nearly inextensible interface so that solving an unknown tension (which enforces the inextensibility constraint) can be avoided. Furthermore, the Fourier spectral method is used to evaluate interfacial derivatives so that we can compute the geometrical quantities on the interface more accurately. The convergence study of inextensible approach can be found in our previous work for axisymmetric vesicles 
[14] and another recent work for fully three-dimensional vesicles [40]. Lastly, as aforementioned, we consider the vesicle electrohydrodynamics in Navier-Stokes flow as opposed to most of existing works for Stokes flow. This enables us to investigate possible inertia effects at finite Reynolds numbers.

The paper is organized as follows. The formulation for the vesicle electrohydrodynamics within the leaky dielectric framework is presented in Section 2. An efficient immersed interface method for solving the electric and transmembrane potentials is introduced and validated in Section 3. The new numerical algorithm that couples immersed boundary and immersed interface method for solving the vesicle electrohydrodynamics is outlined in Section 4. A series of numerical simulations to investigate the dynamics of prolate-oblateprolate transition and the study of how the electric field affects the vesicle dynamics in a shear flow are presented in Section 5. Some concluding remarks and future work are given in Section 6.

\section{Governing equations of vesicle electrohydrodynamics}

In this paper, we consider a vesicle containing a leaky dielectric fluid suspended in another leaky dielectric fluid under a uniform DC electric field $\mathbf{E}_{\infty}=\left(0, E_{\infty}\right)$ as illustrated in Figure 1. Separated by the elastic vesicle membrane $\Sigma$ with a membrane capacitance $C_{m}$ and conductance $G_{m}$, the fluids inside (domain $\Omega^{-}$) and outside (domain $\Omega^{+}$) of the vesicle are characterized by electric conductivity $\sigma$ and permittivity $\varepsilon$. Here, we denote $\sigma^{-}$and $\varepsilon^{-}$as the fluid electric conductivity and permittivity inside the vesicle, and $\sigma^{+}$and $\varepsilon^{+}$for the suspending fluid. The conductivity and permittivity ratios are denoted as $\sigma_{r}=\sigma^{-} / \sigma^{+}$ and $\varepsilon_{r}=\varepsilon^{-} / \varepsilon^{+}$, respectively. The dynamics of the vesicle under a DC electric field is determined by the interaction between the hydrodynamics, elastic bending, tension, and electric stresses on the membrane. For simplicity, we assume that both the fluid density and viscosity are identical for the interior and suspending fluids.
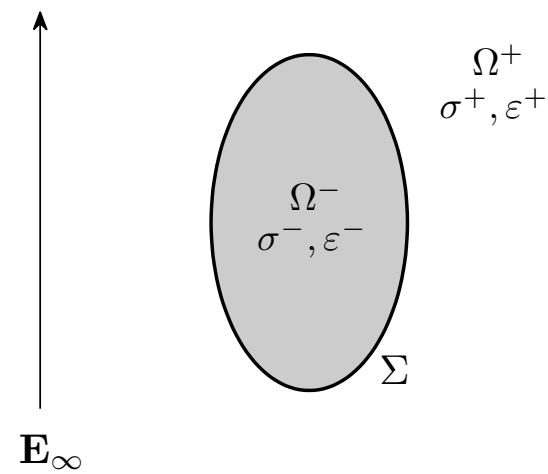

Figure 1: A vesicle suspended in a fluid subject to a uniform DC electric field $\mathbf{E}_{\infty}=\left(0, E_{\infty}\right)$.

The vesicle electrohydrodynamics is governed by the two-dimensional incompressible Navier-Stokes equations with an external DC electric field and forces on the vesicle mem- 
brane. The immersed boundary formulation is an Eulerian-Lagrangian framework in which the fluid variables (such as velocity field, pressure, and electric potential) are defined in the Eulerian manner, while the vesicle membrane related variables (such as membrane position and the electric Maxwell stress) are defined in the Lagrangian manner. For example, the vesicle membrane $\Sigma$ is represented in a Lagrangian parametric form as $\mathbf{X}(s, t)=$ $(X(s, t), Y(s, t)), 0 \leq s \leq 2 \pi$, where $s$ is a parameter of the initial configuration of the immersed boundary (vesicle membrane). Under this representation, the arc-length element is defined as $\left|\mathbf{X}_{s}\right| \mathrm{d} s=\sqrt{X_{s}^{2}+Y_{s}^{2}} \mathrm{~d} s$, where the subscript $s$ denotes the partial derivative with respect to $s$. Thus, the governing equations can be written as a single fluid system in the domain $\Omega=\Omega^{-} \cup \Omega^{+}$

$$
\begin{aligned}
& \rho\left(\frac{\partial \mathbf{u}}{\partial t}+(\mathbf{u} \cdot \nabla) \mathbf{u}\right)=-\nabla p+\mu \Delta \mathbf{u}+\mathbf{f} \quad \text { in } \Omega, \\
& \nabla \cdot \mathbf{u}=0 \quad \text { in } \Omega \\
& \mathbf{f}(\mathbf{x}, t)=\int_{\Sigma}\left(\mathbf{F}_{\gamma}+\mathbf{F}_{b}+\mathbf{F}_{E}\right)(s, t) \delta(\mathbf{x}-\mathbf{X}(s, t))\left|\mathbf{X}_{s}\right| \mathrm{d} s \text { in } \Omega, \\
& \frac{\partial \mathbf{X}}{\partial t}(s, t)=\mathbf{U}(s, t)=\int_{\Omega} \mathbf{u}(\mathbf{x}, t) \delta(\mathbf{x}-\mathbf{X}(s, t)) \mathrm{d} \mathbf{x} \text { on } \Sigma, \\
& \nabla_{s} \cdot \mathbf{U}=0 \text { on } \Sigma,
\end{aligned}
$$

where Eqs. (1)-(2) are the familiar incompressible Navier-Stokes equations with the fluid density $\rho$, the velocity $\mathbf{u}$, the pressure $p$, and the dynamic viscosity $\mu$. The external forcing term $\mathbf{f}$ due to immersed boundary force in Eq. (3) consists of the membrane elastic tension force $\mathbf{F}_{\gamma}$, the bending force $\mathbf{F}_{b}$, and the electric force $\mathbf{F}_{E}$. Eq. (4) simply states that the membrane moves along with the local fluid velocity (the interfacial velocity). The Eulerian fluid and the Lagrangian immersed boundary variables are linked by the two-dimensional Dirac delta function $\delta(\mathbf{x})=\delta(x) \delta(y)$. Eq. (5) describes the inextensibility constraint of the vesicle membrane which is equivalently written as zero surface divergence (defined below) of the velocity along the membrane. Next, we describe the immersed boundary forces in Eq. (3) in detail.

A vesicle lipid bilayer membrane is inextensible and resistant to bending. Thus the total membrane elastic energy $E$ consists of two parts; namely, a surface energy $E_{\gamma}$ that involves the membrane tension arising from the inextensibility constraint, and a Helfrich type energy $E_{b}[9]$ to resist the bending of membrane. The total membrane energy is hence

$$
E(t)=E_{\gamma}(t)+E_{b}(t)=\int_{\Sigma}\left(\gamma+\frac{c_{b}}{2} \kappa^{2}\right)\left|\mathbf{X}_{s}\right| \mathrm{d} s,
$$

where $\gamma$ is the membrane tension (which acts as a Lagrange multiplier) determined from the inextensibility constraint Eq. (5), $c_{b}$ is the bending modulus, and $\kappa$ is the membrane curvature. By taking the variational derivative to the membrane energy in Eq. (6), the vesicle tension force $\mathbf{F}_{\gamma}$ and bending force $\mathbf{F}_{b}$ are obtained as

$$
\mathbf{F}_{\gamma}=\frac{1}{\left|\mathbf{X}_{s}\right|} \gamma_{s} \boldsymbol{\tau}-\gamma \kappa \mathbf{n}, \quad \mathbf{F}_{b}=c_{b}\left(\Delta_{s} \kappa+\frac{\kappa^{3}}{2}\right) \mathbf{n}
$$


where $\boldsymbol{\tau}=\left(\tau_{1}, \tau_{2}\right)=\mathbf{X}_{s} /\left|\mathbf{X}_{s}\right|$ and $\mathbf{n}=\left(\tau_{2},-\tau_{1}\right)$ are the unit tangent and normal vector along the vesicle membrane respectively. The surface divergence operator $\nabla_{s}$. in Eq. (5) and the surface Laplace (or Laplace-Beltrami) operator $\Delta_{s}$ in Eq. (7) are defined, respectively, as

$$
\nabla_{s} \cdot=\frac{1}{\left|\mathbf{X}_{s}\right|} \frac{\partial}{\partial s} \cdot \boldsymbol{\tau} \quad \text { and } \quad \Delta_{s}=\frac{1}{\left|\mathbf{X}_{s}\right|} \frac{\partial}{\partial s}\left(\frac{1}{\left|\mathbf{X}_{s}\right|} \frac{\partial}{\partial s}\right)
$$

The detailed derivation of Eq. (7) can be found in [17]. Notice that, the sign of curvature $\kappa$ is positive for a circle under the current parametric representation.

The electric stress in a dielectric medium under an electric field is given by the Maxwell stress tensor of the form $[29,36]$

$$
\mathbf{M}_{E}=\varepsilon\left(\mathbf{E E}-\frac{1}{2}(\mathbf{E} \cdot \mathbf{E}) I\right)
$$

Since the permittivity $\varepsilon$ and electric field $\mathbf{E}$ are discontinuous across the membrane, we regard the electric effect as a membrane force arising from the jump of the Maxwell tensor as in our previous work for the droplet electrohydrodynamics [15]. That is,

$$
\mathbf{F}_{E}=\left[\mathbf{M}_{E} \cdot \mathbf{n}\right]=\left(\mathbf{M}_{E}^{+}-\mathbf{M}_{E}^{-}\right) \cdot \mathbf{n},
$$

where $\mathbf{M}_{E}^{-}$and $\mathbf{M}_{E}^{+}$stand for Maxwell stress tensor inside the vesicle and surrounding fluid, respectively.

We also note that the same membrane electric force has been used in the boundary integral method [30, 31] and level set method [22] for vesicle electrohydrodynamic simulations. The remaining issue is how to find the electric field $\mathbf{E}$ in the domain $\Omega$, which we address as follows.

\subsection{Vesicle leaky dielectric model}

In this paper we adopt the leaky dielectric model where electric charges in the bulk relax on a much shorter timescale than the characteristic fluid dynamic timescale [36, 41], while the charges are allowed to accumulate on an interface that separates two media with mismatched electrical properties. Accordingly, the electric field $\mathbf{E}$ is irrotational and can be represented by $\mathbf{E}=-\nabla \phi$, where the electric potential $\phi$ satisfies the Laplace equation

$$
\Delta \phi=0 \quad \text { in } \Omega \backslash \Sigma .
$$

Under an electric field the lipid bilayer membrane charges as a capacitor. Opposite charges accumulate on either side of the vesicle membrane and thus form a transmembrane potential $V_{m}$ (unlike the droplet interface behavior, see [15] for example). That is,

$$
[\phi]=\phi^{+}-\phi^{-}=V_{m}(s, t) \quad \text { on } \Sigma,
$$

where the bracket [.] stands for the jump of the quantity approaching from the $\Omega^{+}$side to the $\Omega^{-}$side. Also under the leaky dielectric assumption, the normal component of ohmic current $\mathbf{J}=\sigma \mathbf{E}$ is continuous across the membrane. Thus, we have

$$
\left[\sigma \phi_{n}\right]=\sigma^{+} \phi_{n}^{+}-\sigma^{-} \phi_{n}^{-}=-\left(\mathbf{J}^{+}-\mathbf{J}^{-}\right) \cdot \mathbf{n}=0 \quad \text { on } \Sigma,
$$


where the shorthand $\phi_{n}$ denotes the normal derivative $\nabla \phi \cdot \mathbf{n}$. The transmembrane potential $V_{m}$ is calculated from the conservation law of current density across the membrane [38]

$$
C_{m} \frac{\partial V_{m}}{\partial t}+G_{m} V_{m}=\sigma^{+} \phi_{n}^{+}=\sigma^{-} \phi_{n}^{-} \quad \text { on } \Sigma,
$$

where $C_{m}$ and $G_{m}$ are the membrane capacitance and conductance, respectively. It is worth mentioning that the transmembrane potential is defined by the potential jump from the $\Omega^{-}$ side to the $\Omega^{+}$side so there is a sign difference between the present $V_{m}$ and the one used in [38]. As a result, the righthand side of Eq. (14) in [38] will be $\sigma^{+} \mathbf{E}^{+} \cdot \mathbf{n}=\sigma^{-} \mathbf{E}^{-} \cdot \mathbf{n}$.

\section{An immersed interface method for the bulk electric poten- tial $\phi$ and transmembrane potential $V_{m}$}

The immersed interface method (IIM) is a jump-capturing method on a Cartesian grid for solving elliptic equations with discontinuities across the interfaces, see Li and Ito [26] for more details. In this section, we will develop an augmented immersed interface method for solving the electric potential $\phi$ and the transmembrane potential $V_{m}$. That is, we need to solve Eqs. (11) and (14) simultaneously with the jump conditions Eqs. (12)-(13). Our present numerical scheme is an extension of our previous electric potential solver for a viscous drop [15], where there is no jump in electric potential across the fluid interface (no need to solve Eq. (14) there).

To proceed, let us first layout a uniform Cartesian grid in the computational domain $\Omega$ with mesh width $h=\Delta x=\Delta y$ for simplicity. The grid point $\mathbf{x}_{i j}$ is located at the grid center where the discrete potential $\phi_{i j}=\phi\left(\mathbf{x}_{i j}\right)$ is defined. The interface $\Sigma$ embedded in $\Omega$ cuts through some grid cells so the potential solution is not smooth across the interface as we can see from the jump conditions Eqs. (12)-(13). We thus classify the grid point as either a regular or irregular point: For a regular point, it means that the standard five-point Laplacian discretization (denoted by $\Delta_{h}$ ) for Eq. (11) does not cut through the interface so the second-order local truncation error is achieved. On the other hand, at an irregular point, the five-point Laplacian cuts through the interface so the grid points used involve both inside and outside the interface. Since the solution and its derivatives have jumps across the interface, a correction for the Laplacian discretization is needed at the irregular point to maintain the desired accuracy. Thus, the discretization of Eq. (11) at the grid point $\mathbf{x}_{i j}$ can be generally written in the form of

$$
\Delta_{h} \phi_{i j}=-\frac{C_{i j}}{h^{2}}
$$

where $C_{i j}$ is the correction term which is nonzero only if the grid point is irregular.

Let us describe what the correction term is at the particular irregular point as depicted in Figure 2(a). When we apply the five-point Laplacian to $\mathbf{x}_{i j}$, the grid point $\mathbf{x}_{i-1, j}$ falls in different side of the interface so the correction of discretization comes only from the point $\mathbf{x}_{i-1, j}$. To derive the correction term, one needs to find the orthogonal projection of $\mathbf{x}_{i-1, j}$ at the interface (say $\mathbf{X}_{*}=\mathbf{X}\left(s_{*}\right)$ in Figure 2(a)), and then apply the Taylor's expansion 


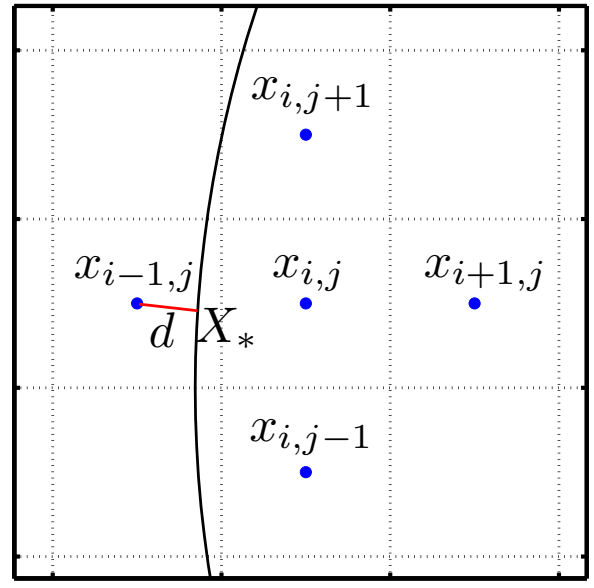

(a)

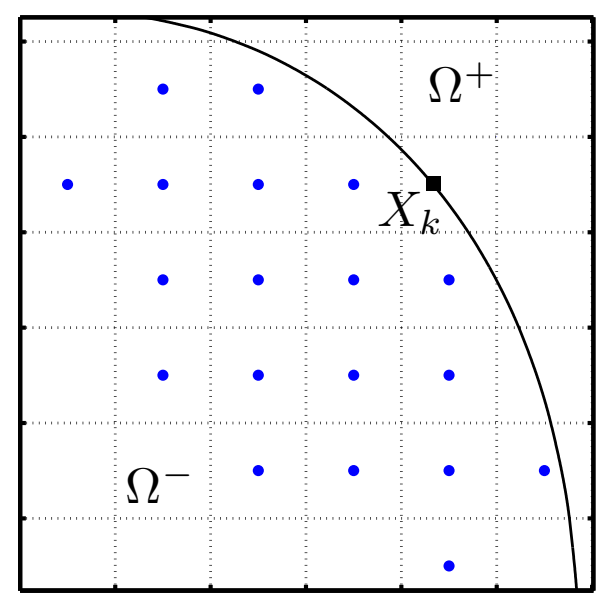

(b)

Figure 2: (a) The five-point Laplacian of the irregular point $\mathbf{x}_{i j}$. (b) A diagram showing least squares approximation for one-sided normal derivative at $\mathbf{X}_{k}$.

along the normal direction at $\mathbf{X}_{*}$. The correction term thus becomes

$$
C_{i j}=[\phi]_{\mathbf{X}_{*}}+d\left[\phi_{n}\right] \mathbf{X}_{*}+\frac{d^{2}}{2}\left(-\kappa_{\mathbf{X}_{*}}\left[\phi_{n}\right] \mathbf{X}_{*}-\Delta_{s}[\phi] \mathbf{X}_{*}\right),
$$

where the value $d$ is the signed distance between the grid point $\mathbf{x}_{i-1, j}$ and the orthogonal projection $\mathbf{X}_{*}$, and $\kappa$ is the local curvature of the interface. The more detailed derivation of the above correction term can be found in [25, 33].

Since the above correction term involves the usage of normal derivative jump $\left[\phi_{n}\right]$, it is quite natural to use the jump condition Eq. (13) to derive the following equation as in $[15,44]$

$$
\phi_{n}^{-}+\frac{\sigma^{+}}{[\sigma]}\left[\phi_{n}\right]=\frac{\left[\sigma \phi_{n}\right]}{[\sigma]}=0 .
$$

Here, we assume that the vesicle is less conductive than the surrounding fluid $\left(\sigma^{-}<\sigma^{+}\right)$. Other formula can be used for the case $\left(\sigma^{-}>\sigma^{+}\right)$as shown in $[15,44]$. The terms in the righthand side of transmembrane potential Eq. (14) involve one-sided normal derivatives $\sigma^{+} \phi_{n}^{+}$and $\sigma^{-} \phi_{n}^{-}$. One can further rewrite the equation separately by

$$
\frac{C_{m}}{\sigma^{+}} \frac{\partial V_{m}}{\partial t}+\frac{G_{m}}{\sigma^{+}} V_{m}=\phi_{n}^{+}, \quad \frac{C_{m}}{\sigma^{-}} \frac{\partial V_{m}}{\partial t}+\frac{G_{m}}{\sigma^{-}} V_{m}=\phi_{n}^{-},
$$

and then subtract the above first equation by the second one to obtain

$$
\left(1-\frac{1}{\sigma_{r}}\right)\left(\frac{C_{m}}{\sigma^{+}} \frac{\partial V_{m}}{\partial t}+\frac{G_{m}}{\sigma^{+}} V_{m}\right)=\left[\phi_{n}\right] \quad \text { on } \Sigma,
$$

where the conductivity ratio is defined by $\sigma_{r}=\sigma^{-} / \sigma^{+}$. The advantage of using the above Eq. (18) instead of Eq. (14) is to establish the relationship between the two unknown 
interfacial functions $V_{m}=[\phi]$ and $\left[\phi_{n}\right]$. This new formulation simplifies our numerical algorithm as we can see later. McConnell et. al. exploited the similar formulation for the boundary integral method to simulate vesicle electrohydrodynamics in Stokes regime [30].

\subsection{Implementation details}

We now write down the discretization details for Eqs. (15)-(18), and solve the resultant matrix equation. Since the vesicle membrane is self-enclosing, we can use the Fourier spectral discretization to represent the configuration $\mathbf{X}$. We first choose a collection of discrete Lagrangian markers $\mathbf{X}_{k}=\mathbf{X}\left(s_{k}\right)$ with equally distributed interfacial coordinates $s_{k}=k \Delta s, k=0,1, \cdots, M$ with $\Delta s=2 \pi / M$. The membrane thus can be represented in truncated Fourier series (in vector form) as

$$
\mathbf{X}(s)=\sum_{l=-M / 2}^{M / 2-1} \widehat{\mathbf{X}}_{l} e^{i l s},
$$

where $\widehat{\mathbf{X}}_{l}$ are the Fourier coefficients for $\mathbf{X}(s)$, and can be computed very efficiently using the Fast Fourier Transform (FFT). Under this Fourier representation, the derivatives with respect to $s$ can also be computed quite easily by using the pseudospectral method [42]. In this fashion, all geometrical quantities such as the tangent vector $\tau$ (thus, the normal vector $\mathbf{n}$ ) and the curvature $\kappa$ can be computed with spectral accuracy. As noted before, all the vesicle membrane variables are defined in Lagrangian manner so the jumps of $[\phi]=V_{m}$ and $\left[\phi_{n}\right]$ are all defined at $\mathbf{X}_{k}$. Thanks to the Fourier representation, the jumps $[\phi]$ and $\left[\phi_{n}\right]$ at the orthogonal projection points $\mathbf{X}_{*}$ in the correction term in Eq. (16) can be easily interpolated through the values at the markers $\mathbf{X}_{k}$. Meanwhile, the curvature $\kappa_{\mathbf{X}_{*}}$ and $\Delta_{s}[\phi]_{\mathbf{X}_{*}}$ in Eq. (16) can be also computed in a spectral manner. One should mention that the present representation for the unknown derivative jump $\left[\phi_{n}\right]$ defined at the marker points $\mathbf{X}_{k}$ differs from our previous work on the droplet case [15] in which $\left[\phi_{n}\right]$ is defined directly at those projection points $\mathbf{X}_{*}$.

Let $\Phi, \Psi_{1}$ and $\Psi_{2}$ be the solution vectors formed by $\phi_{i j},[\phi]_{\mathbf{X}_{k}}$ and $\left[\phi_{n}\right]_{\mathbf{X}_{k}}$, then the difference Eq. (15) can be alternatively written in the matrix form of

$$
A \Phi+C_{1} \Psi_{1}+C_{2} \Psi_{2}=F
$$

where $C_{1}$ and $C_{2}$ are the formal matrix operators involving the Fourier interpolation of jumps in the correction term as described in the previous subsection. The righthand side vector $F$ simply results from the boundary conditions for the potential $\phi=-E_{\infty} y$. To be complete, we need two more matrix equations for $\Psi_{1}$ and $\Psi_{2}$, respectively.

As discussed earlier, the value of the normal derivative jump $\left[\phi_{n}\right] \mathbf{x}_{k}$ (denoted by the vector $\Psi_{2}$ ) can be linked to the known jump $\left[\sigma \phi_{n}\right] \mathbf{x}_{k}$ through Eq. (17). However, as we can see from Eq. (17), a one-sided normal derivative approximation $\phi_{n}^{-}\left(\mathbf{X}_{k}\right)$ is needed. To approximate that, we first construct a least squares cubic polynomial by using the values of $\phi_{i j}$ at the grid points satisfying $\left\|\mathbf{x}_{i j}-\mathbf{X}_{k}\right\| \leq 4 h$ in the domain $\Omega^{-}$as depicted in Figure 2(b). In general, the number of chosen grid points is more than the number of unknown coefficients in the least squares approximation so the cubic polynomial $P(x, y)$ 
can be obtained. For the interface point $\mathbf{X}_{k}$ in a high curvature region, we have to increase the spatial resolution to make sure enough grid points fall into the same region so the least squares cubic polynomial is achievable. Once we have the polynomial $P(x, y)$, the one-sided normal derivative $\phi_{n}^{-}\left(\mathbf{X}_{k}\right)$ can be computed by $\nabla P\left(\mathbf{X}_{k}\right) \cdot \mathbf{n}\left(\mathbf{X}_{k}\right)$ directly. This least squares cubic polynomial approach roughly has third-order of accuracy to the approximation of the function itself while the derivative has second-order of accuracy. With this approximation, we can rewrite Eq. (17) in a matrix form as

$$
B^{-} \Phi+\frac{\sigma^{+}}{[\sigma]} \Psi_{2}=0
$$

where $B^{-}$denotes the resultant matrix from the above least squares cubic polynomial approximation formally.

Now we need to construct the matrix equation for the unknown vector $\Psi_{1}$ which the transmembrane potential Eq. (18) should be used. Here, we provide the Backward Euler $(\mathrm{BE})$ and the Crank-Nicholson $(\mathrm{CN})$ method for the time integration as follows.

- BE:

$$
\left(1-\frac{1}{\sigma_{r}}\right)\left(\frac{C_{m}}{\sigma^{+}} \frac{\Psi_{1}-\Psi_{1}^{n}}{\Delta t}+\frac{G_{m}}{\sigma^{+}} \Psi_{1}\right)=\Psi_{2}
$$

- $\mathrm{CN}$ :

$$
\left(1-\frac{1}{\sigma_{r}}\right)\left(\frac{C_{m}}{\sigma^{+}} \frac{\Psi_{1}-\Psi_{1}^{n}}{\Delta t}+\frac{G_{m}}{\sigma^{+}} \frac{\Psi_{1}+\Psi_{1}^{n}}{2}\right)=\frac{\Psi_{2}+\Psi_{2}^{n}}{2} .
$$

Here, $\Psi_{1}^{n}$ and $\Psi_{2}^{n}$ are given in the previous time step with the time step size $\Delta t$.

We can couple the matrix Eqs. (20)-(21) with either Eq. (22) (BE) or Eq. (23) (CN) into one linear system as

$$
\left[\begin{array}{ccc}
A & C_{1} & C_{2} \\
B^{-} & 0 & \frac{\sigma^{+}}{[\sigma]} I \\
0 & \alpha I & \beta I
\end{array}\right]\left[\begin{array}{c}
\Phi \\
\Psi_{1} \\
\Psi_{2}
\end{array}\right]=\left[\begin{array}{c}
F \\
0 \\
G
\end{array}\right]
$$

where $\alpha$ and $\beta$ are nonzero (if $\sigma_{r} \neq 1$ ) constant coefficients depending on the usage of Eq. (22) or (23), and the vector $G$ collects the known terms. In practice, we do not form the matrices $C_{1}, C_{2}$ and $B^{-}$explicitly as we can see from the iterative procedure of our matrix solver below. To solve the above linear system, we first eliminate $\Phi$ and $\Psi_{2}$ from Eq. (24) by Schur complement technique (after some careful matrix calculations) to obtain a new linear system of $\Psi_{1}$ as

$$
\left(B^{-} A^{-1}\left(C_{1}-\frac{\alpha}{\beta} C_{2}\right)+\frac{\alpha}{\beta} \frac{\sigma^{+}}{[\sigma]} I\right) \Psi_{1}=B^{-} A^{-1}\left(F-\frac{1}{\beta} C_{2} G\right)+\frac{1}{\beta} \frac{\sigma^{+}}{[\sigma]} G .
$$

This is a $M \times M$ system for $\Psi_{1}$ which is much smaller than the original one. We then use the GMRES iterative method to solve the above linear system. Since the GMRES method 
only requires the matrix-vector multiplication, it is not necessary to construct the matrices $A^{-1}, B^{-}, C_{1}$ and $C_{2}$ explicitly. Note that, the matrix $A$ comes from the five-point Laplacian discretization so the inversion of $A$ can be performed efficiently by applying the fast Poisson solver provided by Fishpack public software package [1].

In summary, the detailed numerical algorithm for solving the linear system Eq. (24) to find $\Phi, \Psi_{1}$ and $\Psi_{2}$ can be split into the following four steps.

Step 1. Apply one fast Poisson solver to obtain $\Phi^{*}$ in

$$
A \Phi^{*}=F-\frac{1}{\beta} C_{2} G .
$$

Step 2. Use GMRES iteration to solve $\Psi_{1}$ in

$$
\left(B^{-} A^{-1}\left(C_{1}-\frac{\alpha}{\beta} C_{2}\right)+\frac{\alpha}{\beta} \frac{\sigma^{+}}{[\sigma]} I\right) \Psi_{1}=B^{-} \Phi^{*}+\frac{1}{\beta} \frac{\sigma^{+}}{[\sigma]} G .
$$

Step 3. Update $\Psi_{2}$ explicitly by Eq. (22) or (23).

Step 4. Apply one fast Poisson solver to solve $\Phi$ in

$$
A \Phi=F-C_{1} \Psi_{1}-C_{1} \Psi_{2}
$$

During the GMRES iteration in Step 2, we set the stopping criterion as $h^{2}$ which has the same magnitude as the local truncation error of our scheme. The overall computational cost for Step 1-4 in our present scheme can be evaluated in terms of the number of fast Poisson solver being applied.

Let us conclude this subsection by remarking the comparison between the present numerical algorithm and the one proposed by Kolahdouz and Salac [21] for solving the above electric and transmembrane potential equations. Although both algorithms share the same IIM spirit developed earlier in [25, 33], the authors in [21] use the one-sided normal derivative $\phi_{n}^{-}$as the unknowns in GMRES iterations while here we use the normal derivative jump of $\left[\phi_{n}\right]$ as the unknowns directly. Meanwhile, in [21], the surface Laplacian jump $\Delta_{s}[\phi]$ at the projection point needed in the correction term of Eq. (16) is obtained by first extending the jump $[\phi]=V_{m}$ along the normal direction to the grid points via closest point method [28] with level set representation of the interface and then evaluating the standard Cartesian Laplacian $\Delta[\phi]$ (equals to $\Delta_{s}[\phi]$ ) on the grid points. However, the present Fourier spectral representation with Lagrangian markers is able to calculate $\Delta_{S}[\phi]$ directly. In the next subsection, we shall provide a more systematic numerical check on the accuracy and efficiency for our present numerical algorithm.

\subsection{Numerical test: accuracy and efficiency study}

In this subsection, we demonstrate the accuracy and efficiency test for the numerical scheme of the IIM for solving the electric and transmembrane potentials developed in the previous 
subsection. All the numerical computations were carried out on a desktop PC with Intel Core i7 CPU and 16G RAM.

Example 1. We construct an analytical solution for Eqs. (11) and (14) with the jump conditions Eqs. (12)-(13) on a circular interface $\mathbf{X}(s)=(0.5 \cos s, 0.5 \sin s)$ as

$$
\phi(x, y, t)=\left\{\begin{array}{ll}
\frac{e^{-t}}{\sigma^{-}}\left(x^{2}-y^{2}\right) & \mathbf{x} \in \Omega^{-} \\
\frac{e^{-t}}{\sigma^{+}}\left(x^{2}-y^{2}\right) & \mathbf{x} \in \Omega^{+}
\end{array}, \quad V_{m}(s, t)=\left(1-\frac{1}{\sigma_{r}}\right) \frac{e^{-t}}{\sigma^{+}}\left(X(s)^{2}-Y(s)^{2}\right) .\right.
$$

The computational domain is chosen as $\Omega=[-1,1] \times[-1,1]$. The conductivities are chosen as $\sigma^{-}=0.1, \sigma^{+}=1\left(\sigma_{r}=0.1\right)$. The membrane capacitance and conductance are $C_{m}=1$ and $G_{m}=\frac{4}{1-\sigma_{r}}+C_{m}$, respectively. We set $N$ to be the grid size and thus the mesh size is $h=2 / N$. The time step for the discretization of transmembrane potential Eq. (22) or (23) is chosen in the same order of mesh width as $\Delta t=h / 4$ and the simulation is ran up to $T=0.2$. We present the rate of convergence for the maximum error between the numerical solution $\phi_{h}$ and the exact solution $\phi_{e}$ in Table 1. As expected, the first- and second-order convergence are achieved for $\mathrm{BE}$ and $\mathrm{CN}$ scheme, respectively. As for the computational complexity, one can see that the average number of GMRES iterations in the time interval $[0,0.2]$ is less than 4 and becomes steady even as the grid number doubles.

\begin{tabular}{lccccccc}
\hline \hline \multirow{2}{*}{$N$} & \multicolumn{3}{c}{$\mathrm{BE}$} & & \multicolumn{3}{c}{$\mathrm{CN}$} \\
\cline { 2 - 4 } \cline { 7 - 8 } & $\left\|\phi_{h}-\phi_{e}\right\|_{\infty}$ & Rate & Iter. & & $\left\|\phi_{h}-\phi_{e}\right\|_{\infty}$ & Rate & Iter. \\
\hline 80 & $8.046 \mathrm{E}-04$ & - & 3 & & $2.128 \mathrm{E}-04$ & - & 3 \\
160 & $4.958 \mathrm{E}-04$ & 0.69 & 3 & & $3.168 \mathrm{E}-05$ & 2.74 & 4 \\
320 & $2.587 \mathrm{E}-04$ & 0.93 & 3 & & $7.959 \mathrm{E}-06$ & 1.99 & 4 \\
640 & $1.313 \mathrm{E}-04$ & 0.97 & 3 & & $1.994 \mathrm{E}-06$ & 2.00 & 4 \\
\hline \hline
\end{tabular}

Table 1: Mesh refinement results for accuracy and efficiency for Example 1. The numerical solution is denoted by $\phi_{h}$ and exact solution by $\phi_{e}$.

Example 2. In this test, we construct another analytic solution for Eqs. (11)-(13) on a more complicated interface $\mathbf{X}(s)=(r(s) \cos s, r(s) \sin s)$ with the radius function $r(s)=0.1(4+\cos 3 s)$ (see Figure 3$)$ as

$$
\phi(x, y, t)=\left\{\begin{array}{ll}
\frac{e^{-t}}{\sigma^{-}} e^{x} \cos y & \mathbf{x} \in \Omega^{-} \\
\frac{e^{-t}}{\sigma^{+}} e^{x} \cos y & \mathbf{x} \in \Omega^{+}
\end{array}, \quad V_{m}(s, t)=\left(1-\frac{1}{\sigma_{r}}\right) \frac{e^{-t}}{\sigma^{+}} e^{X(s)} \cos Y(s) .\right.
$$

Again the computational domain is $\Omega=[-1,1] \times[-1,1]$. We use the same conductivities as Example 1 but with a capacitance of $C_{m}=1$ and conductance of $G_{m}=0.1$. However, the transmembrane potential $V_{m}$ does not satisfy Eq. (18) exactly so an extra term must be added in the righthand side of the equation. The grid size and the time step are the same as the ones in Example 1 and the solution is computed up to $T=0.2$. Table 2 shows the mesh refinement analysis for our numerical results. Again, we obtain the corresponding first and second-order convergence for BE and CN scheme and the average number of GMRES 
iterations still stays steady as we double the grid size $N$. This numerical test demonstrates the robust capability of our present algorithm to handle an interface with more complex geometry.

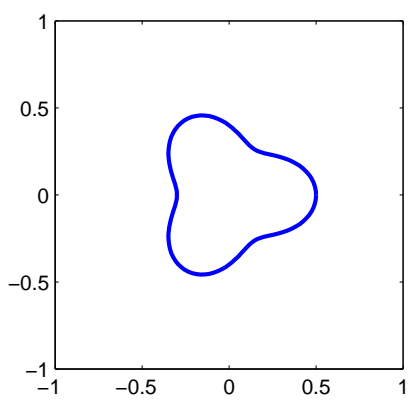

Figure 3: Membrane shape $\mathbf{X}(s)=(r(s) \cos s, r(s) \sin s)$ with the radius function $r(s)=$ $0.1(4+\cos 3 s)$.

\begin{tabular}{lccccccc}
\hline \hline \multirow{2}{*}{$N$} & \multicolumn{3}{c}{$\mathrm{BE}$} & & \multicolumn{3}{c}{$\mathrm{CN}$} \\
\cline { 2 - 4 } \cline { 7 - 8 } \cline { 6 - 8 } & $\left\|\phi_{h}-\phi_{e}\right\|_{\infty}$ & Rate & Iter. & & $\left\|\phi_{h}-\phi_{e}\right\|_{\infty}$ & Rate & Iter. \\
\hline 80 & $8.299 \mathrm{E}-03$ & - & 8 & & $7.249 \mathrm{E}-04$ & - & 9 \\
160 & $4.033 \mathrm{E}-03$ & 1.04 & 9 & & $6.313 \mathrm{E}-05$ & 3.52 & 10 \\
320 & $2.017 \mathrm{E}-03$ & 0.99 & 9 & & $1.229 \mathrm{E}-05$ & 2.36 & 9 \\
640 & $1.010 \mathrm{E}-03$ & 0.99 & 8 & & $3.204 \mathrm{E}-06$ & 1.93 & 8 \\
\hline \hline
\end{tabular}

Table 2: Mesh refinement results for accuracy and efficiency for Example 2. The numerical solution is denoted by $\phi_{h}$ and exact solution is by $\phi_{e}$.

\section{Numerical algorithm}

The governing equations (1)-(5), (7), (10), (11) and (14) are rendered non-dimensional as follows. We use the effective radius of vesicle $R=\sqrt{A_{0} / \pi}$ as a scaling length, where $A_{0}$ is the enclosed area of vesicle. We use the membrane charging time of a quasi-spherical non-conducting membrane [16] $t_{m m}=\frac{R C_{m}}{\sigma^{+}}\left(\frac{1}{2}+\frac{1}{\sigma_{r}}\right)$ as our characteristic timescale. All physical variables are scaled by the associated characteristic scales in the following,

$$
\mathbf{x}^{*}=\frac{\mathbf{x}}{R}, \quad t^{*}=\frac{t}{t_{m m}}, \quad p^{*}=\frac{t_{m m}^{2}}{\rho R^{2}} p, \quad \gamma^{*}=\frac{R^{2}}{c_{b}} \gamma, \quad \varepsilon^{*}=\frac{\varepsilon}{\varepsilon^{+}}, \quad \sigma^{*}=\frac{\sigma}{\sigma^{+}}, \quad \mathbf{E}^{*}=\frac{\mathbf{E}}{E_{\infty}} .
$$


The dimensionless governing equations for the vesicle electrohydrodynamics (after dropping $*$ in notations) can be summarized as

$$
\begin{aligned}
& \frac{\partial \mathbf{u}}{\partial t}+(\mathbf{u} \cdot \nabla) \mathbf{u}=-\nabla p+\frac{1}{R e} \Delta \mathbf{u} \\
& +\int_{\Sigma}\left(\mathbf{F}_{\gamma}+\frac{1}{R e C a} \mathbf{F}_{b}+\frac{M n}{R e} \mathbf{F}_{E}\right) \delta(\mathbf{x}-\mathbf{X}(s, t))\left|\mathbf{X}_{s}\right| \mathrm{d} s \text { in } \Omega, \\
& \nabla \cdot \mathbf{u}=0 \quad \text { in } \Omega, \\
& \frac{\partial \mathbf{X}}{\partial t}(s, t)=\mathbf{U}(s, t)=\int_{\Omega} \mathbf{u}(\mathbf{x}, t) \delta(\mathbf{x}-\mathbf{X}(s, t)) \mathrm{d} \mathbf{x} \text { on } \Sigma,
\end{aligned}
$$

where

$$
\begin{aligned}
& \gamma=\gamma_{0}\left(\left|\mathbf{X}_{s}\right|-\left|\mathbf{X}_{s}\right|^{0}\right) \quad \text { on } \Sigma \\
& \mathbf{F}_{\gamma}=\frac{1}{\left|\mathbf{X}_{s}\right|} \gamma_{s} \boldsymbol{\tau}-\gamma \kappa \mathbf{n}, \quad \mathbf{F}_{b}=\left(\Delta_{s} \kappa+\frac{\kappa^{3}}{2}\right) \mathbf{n} \quad \text { on } \Sigma \\
& \Delta \phi=0 \quad \text { in } \Omega \backslash \Sigma, \quad[\phi]=V_{m}, \quad\left[\sigma \phi_{n}\right]=0 \quad \text { on } \Sigma \\
& \left(1-\frac{1}{\sigma_{r}}\right)\left(C \frac{\partial V_{m}}{\partial t}+G V_{m}\right)=\left[\phi_{n}\right] \quad \text { on } \Sigma, \\
& \mathbf{E}=-\nabla \phi, \quad \mathbf{M}_{E}=\varepsilon\left(\mathbf{E E}-\frac{1}{2}(\mathbf{E} \cdot \mathbf{E}) I\right), \quad \mathbf{F}_{E}=\left(\mathbf{M}_{E}^{+}-\mathbf{M}_{E}^{-}\right) \cdot \mathbf{n} .
\end{aligned}
$$

Notice that, as our previous work on vesicle dynamics [14, 40], we relax the inextensibility constraint Eq. (5) of vesicle membrane and replace the unknown tension by a spring-like tension as shown in Eq. (29) with a large elastic stiffness $\gamma_{0}$. Here $\left|\mathbf{X}_{s}\right|^{0}$ is the local stretching factor of the vesicle initial configuration. The spring-like tension is intended to keep the stretching factor $\left|\mathbf{X}_{s}\right|$ close to its initial configuration so the current vesicle membrane is nearly inextensible. The numerical validation and convergence of this nearly inextensible approach have been performed in our recent works on 3D axisymmetric [14] and fully 3D vesicle dynamics [40].

There are several dimensionless numbers; namely, the Reynolds number, $R e=\frac{\rho R^{2}}{\mu t_{m m}}$; the capillary number, $C a=\frac{\mu R^{3} / c_{b}}{t_{m m}}$, measures the ratio of restoring bending and membrane charging timescale; the Mason number, $M n=\frac{t_{m m}}{\mu / \varepsilon^{+} E_{\infty}^{2}}$ measures the strength of the electric field; the dimensionless membrane capacitance, $C=\frac{R C_{m} / \sigma^{+}}{t_{m m}}$ measures the membrane charging rate; the dimensionless membrane conductivity, $G=R G_{m} / \sigma^{+}$measures the ratio of charging and membrane conductivity rate. For $2 \mathrm{D}$ vesicle, another dimensionless parameter to measure the deflation of its shape is called the reduced area $\nu=4 \pi A_{0} / L_{0}^{2}$, where $L_{0}$ is the perimeter of vesicle. The dimensionless number is nothing but the area ratio of the vesicle to a circle with the same perimeter. The typical values observed from the experimental data $[31,37,39]$ are as follows: the vesicle radius $R \approx 10^{-5} \mathrm{~m}$, the strength of electric field $E_{\infty} \approx 10^{5} \mathrm{~V} / \mathrm{m}$, the fluid viscosity $\mu \approx 10^{-3} \mathrm{~Pa} \mathrm{~s}$, the fluid density $\rho \approx 10^{3}$ $\mathrm{kg} / \mathrm{m}^{3}$, the bulk fluid conductivity $\sigma^{+} \approx 10^{-4} \mathrm{~S} / \mathrm{m}$, the permittivity $\varepsilon^{+} \approx 10^{-10} \mathrm{~F} / \mathrm{m}$, and 
the membrane conductivity $C_{m} \approx 10^{-2} \mathrm{~F} / \mathrm{m}^{2}$. Referring the above physical parameters and taking $\sigma_{r}=0.1$, we obtain the dimensionless numbers $R e=0.02, M n=20$, and $C=0.1$. Throughout this paper, we set the capillary number $C a=10$ corresponding to $c_{b}=10^{-17} \mathrm{~J}$ (typically $c_{b}=10^{-19} \mathrm{~J}$ ) since larger bending force can stabilize the vesicle shape in present simulations during POP transition without wrinkling as we shall explain later.

Next we describe the numerical scheme to simulate the full vesicle electrohydrodynamic systems Eqs. (26)-(33). As our droplet work in [15], the idea is to solve the fluid equations by the usual immersed boundary method and the electric potential by the immersed interface method proposed in Subection 3.1. We consider the computational domain as a rectangle $\Omega=[a, b] \times[c, d]$. Within this domain, a uniform Cartesian grid with mesh width $h$ in both $x$ and $y$ directions is employed. The fluid variables are defined on the standard staggered marker-and-cell (MAC) manner [8]. That is, the velocity component $u$ and $v$ are defined at the cell-normal edges $\left(x_{i-1 / 2}, y_{j}\right)=(a+(i-1) h, c+(j-1 / 2) h)$ and $\left(x_{i}, y_{j-1 / 2}\right)=(a+(i-$ $1 / 2) h, c+(j-1) h)$ respectively, while the pressure $p$ and the electric potential $\phi$ are both defined at the cell center $\left(x_{i}, y_{j}\right)=(a+(i-1 / 2) h, c+(j-1 / 2) h)$. As mentioned before, for the vesicle membrane interface, we use a spectral collection points $s_{k}=k \Delta s, k=0,1, \ldots M$ with $\Delta s=2 \pi / M$ to represent the Lagrangian markers $\boldsymbol{X}_{k}=\boldsymbol{X}\left(s_{k}\right)$ so that any spatial derivatives can be performed spectrally accurate by using Fast Fourier Transform (FFT). In our previous work [14], it has been confirmed that using the Fourier spectral method to compute interfacial derivatives indeed outperforms the finite difference method used in traditional IB method in terms of accuracy. This accuracy effect is especially profound in the calculation of $\Delta_{s} \kappa$ in the bending force $\mathbf{F}_{b}$ since the fourth-order derivative is involved. Here the pseudospectral method is used to increase the accuracy of the computation of geometrical quantities on vesicle membrane. However, the overall convergence rate of the fluid variables would remain only first-order accurate due to the applied immersed boundary method for the fluid solver.

The time-stepping for the overall vesicle electrohydrodynamic system can be described in the following. At the beginning of each time step $n$, the fluid velocity $\mathbf{u}^{n}$, the vesicle membrane configuration $\mathbf{X}^{n}$, and the transmembrane potential $V_{m}^{n}$ must be given. The detailed numerical algorithm is given as follows.

1. Solve the electric potential $\phi^{n}$ and the transmembrane potential (using the CrankNicholson scheme) by the immersed interface method proposed in Subsection 3.1. Then we perform the one-sided difference by least squares polynomial approach introduced in Subsection 3.1 to compute $\mathbf{E}^{n}=\left(-\phi_{x}^{n},-\phi_{y}^{n}\right)$ at the Lagrangian markers and use them to compute the Maxwell stress tensor $\mathbf{M}_{E}^{+}$and $\mathbf{M}_{E}^{-}$to obtain the interfacial electric force $\mathbf{F}_{E}^{n}$ (also at Lagrangian markers).

2. For the given vesicle membrane configuration $\mathbf{X}^{n}$, we compute the tension force $\mathbf{F}_{\gamma}^{n}$ associated with the spring-like tension $\gamma^{n}=\gamma_{0}\left(\left|\mathbf{X}_{s}\right|^{n}-\left|\mathbf{X}_{s}\right|^{0}\right)$ and the bending force $\mathbf{F}_{b}^{n}$ in Eq. (30).

3. Distribute the interfacial force terms $\mathbf{F}_{E}^{n}, \mathbf{F}_{\gamma}^{n}$, and $\mathbf{F}_{b}^{n}$ from the Lagrangian markers to the fluid grid points by using the discrete delta function as in traditional IB method. Here, the discrete delta function is chosen as the smoothed 4-point piecewise function 
in [45] which has better suppression of non-physical oscillations in the volume force computations.

4. Solve the Navier-Stokes equations by the pressure-increment projection method to obtain new velocity field $\mathbf{u}^{n+1}$.

5. Interpolate the new velocity $\mathbf{u}^{n+1}$ on the fluid grid points to the Lagrangian markers and then move the markers to new position $\mathbf{X}^{n+1}$ by advancing Eq. (28).

\section{$5 \quad$ Numerical results}

In this section we perform a series of numerical simulations for vesicle electrohydrodynamic system. We first check the convergence of the fluid variables and vesicle configuration for our numerical algorithm. Then, we demonstrate the characteristic prolate-oblate-prolate (POP) transition for a vesicle under influence of electric field. We also study how the POP transition is affected by different membrane conductance and Reynolds number. Lastly, we study the vesicle dynamics in electric field with shear flow.

Throughout this paper, we initially put a prolate vesicle with fixed effective radius $R=1$ at the center of the computational domain $\Omega=[-4,4] \times[-4,4]$. The initial velocity field is set to be zero everywhere (except the one for shear flow in later subsection) so the flow is simply driven by the electric field. In most tests, the no-slip boundary condition is imposed on the flow field so that $\mathbf{u}_{b}=\mathbf{0}$ on $\partial \Omega$; for the shear flow case we set $\mathbf{u}_{b}=(\chi y, 0)$ where $\chi$ is the dimensionless shear rate. The external electric field is chosen as $\mathbf{E}_{\infty}=(0,1)$ so the boundary conditions for the potential are $\phi=-y$ (Dirichlet) at $y= \pm 4$, and $\frac{\partial \phi}{\partial x}=0$ (Neumann) at $x= \pm 4$. The mesh width is $h=8 / N$ with the grid size $N$. We choose the number of Lagrangian marker size $M$ so that $\Delta s=2 \pi / M<h$. In the present method, we compute the immersed boundary force at the beginning of each time step so a restrictive time step size constraint is needed to ensure the numerical stability. Since the elastic stiffness number $\gamma_{0}$ must be chosen sufficiently large to guarantee the nearly inextensibility of the vesicle, the time step size is mainly dominated by the choice of $\gamma_{0}$. As discussed in our previous work in [14], we choose the time step size based on the following heuristic constraint $\Delta t=\widetilde{C} h / \sqrt{\gamma_{0}}$, where $\widetilde{C}$ is a number of $O(1)$. Here, we set the elastic number $\gamma_{0}=2 \times 10^{5}$ to make sure the change of vesicle membrane perimeter is within $1 \%$ in all simulations. The time step size is therefore chosen as $\Delta t=h / 100$. Practically, the average number of GMRES iterations in each step is just within 5 steps in most cases.

Unless otherwise stated, we choose the reduced area $\nu=0.9$, the Reynolds number $R e=0.02$, the capillary number $C a=10$, the conductivity ratio $\sigma_{r}=0.1$, the permittivity ratio $\varepsilon_{r}=1$, the membrane capacitance $C=0.1$, and the Mason number $M n=20$.

\subsection{Convergence test for the fluid variables}

As the first test, we perform the convergence check for the fluid variables and vesicle configuration for the present numerical algorithm. We choose the capillary number $C a=1$ and membrane conductance $G=0.05$ with different grid sizes $N=80,160,320,640$. Since the analytical solutions are not available in this test, we compute the errors between two 
successive grids denoted by $\left\|u_{2 N}-u_{N}\right\|$ so that the rate of convergence can be computed as Rate $=\log _{2} \frac{\left\|u_{N}-u_{N / 2}\right\|}{\left\|u_{2 N}-u_{N}\right\|}$. The errors of other variables are computed in the same manner. Notice that, the fluid variables are defined at the staggered grid so when we refine the mesh, the numerical solutions will not coincide with the same grid locations and thus a simple interpolation is implemented. Table 3 shows the maximum errors for the fluid velocity field and the vesicle configuration at different times $T=0.01$ and 0.1 . One can see that the numerical result at $T=0.01$ shows roughly first-order accuracy as traditional IB method while the result at $T=0.1$ shows better than first-order accurate. We attribute this latter outperformance to the smoothness of the computed solutions at $T=0.1$.

Meanwhile, we also check the volume (area in 2D) loss of the vesicle in our present computations. From Table 3 (last column), one can see that the relative volume loss is less than order of magnitude $10^{-5}$ which is negligibly small in present simulations. Very recently, Boyce Griffith [7] has performed rather detailed numerical tests on volume-conservation for different IB implementations. In this work, the pressure-increment projection method under staggered grid discretization is adopted for our fluid solver so it has rather better volume conservation [7].

\begin{tabular}{|c|c|c|c|c|c|c|c|}
\hline$N$ & $\left\|u_{2 N}-u_{N}\right\|_{\infty}$ & Rate & $\left\|v_{2 N}-v_{N}\right\|_{\infty}$ & Rate & $\left\|\mathbf{X}_{2 N}-\mathbf{X}_{N}\right\|_{\infty}$ & Rate & $\frac{\left|A_{N}-A_{0}\right|}{A_{0}}$ \\
\hline 80 & $6.751 \mathrm{E}-02$ & - & $8.857 \mathrm{E}-02$ & - & $6.774 \mathrm{E}-04$ & - & $6.352 \mathrm{E}-06$ \\
\hline 160 & $2.574 \mathrm{E}-02$ & 1.39 & $4.305 \mathrm{E}-02$ & 1.04 & $2.028 \mathrm{E}-04$ & 1.74 & $3.649 \mathrm{E}-06$ \\
\hline 320 & $1.059 \mathrm{E}-02$ & 1.28 & $1.582 \mathrm{E}-02$ & 1.44 & $1.005 \mathrm{E}-04$ & 1.01 & $1.567 \mathrm{E}-06$ \\
\hline
\end{tabular}

\begin{tabular}{|c|c|c|c|c|c|c|c|}
\hline$N$ & $\left\|u_{2 N}-u_{N}\right\|_{\infty}$ & Rate & $\left\|v_{2 N}-v_{N}\right\|_{\infty}$ & Rate & $\left\|\mathbf{X}_{2 N}-\mathbf{X}_{N}\right\|_{\infty}$ & Rate & $\frac{\left|A_{N}-A_{0}\right|}{A_{0}}$ \\
\hline 80 & $1.459 \mathrm{E}-01$ & - & $1.859 \mathrm{E}-01$ & - & $1.006 \mathrm{E}-02$ & - & $9.737 \mathrm{E}-05$ \\
\hline 160 & $4.407 \mathrm{E}-02$ & 1.73 & $5.904 \mathrm{E}-02$ & 1.65 & $3.065 \mathrm{E}-03$ & 1.72 & $2.090 \mathrm{E}-05$ \\
\hline 320 & $1.228 \mathrm{E}-02$ & 1.84 & $1.650 \mathrm{E}-02$ & 1.84 & $8.545 \mathrm{E}-04$ & 1.84 & $7.656 \mathrm{E}-06$ \\
\hline
\end{tabular}

Table 3: Mesh refinement analysis for the velocity component $u$ and $v$, the vesicle shape $\mathbf{X}$, and the relative volume loss of the vesicle at $T=0.01$ and 0.1 .

\subsection{The prolate-oblate-prolate transition}

The prolate-oblate-prolate (POP) transition of a vesicle with $\sigma_{r}<1$ (the encapsulated fluid is less conductive than the suspending fluid) under a DC electric field has been observed in various numerical simulations $[22,30,37,39]$. To validate the proposed numerical scheme, we set the conductance $G=0$ (no ions are allowed to leak across two sides of vesicle membrane). Since the permittivity ratio $\varepsilon_{r}=1$, we simply set $\varepsilon^{-}=\varepsilon^{+}=1$.

The time-evolutional shapes of vesicle are presented in Figure 4. From $T=0$ to $T=0.6$ we see that the vesicle experiences a prolate-oblate transition, and then the vesicle tends 
to recover to a prolate shape and finally attains an equilibrium prolate shape at $T=5$. Note that, the "squaring" shapes of vesicle are observed at $\sim T=0.4$ during the transition between prolate and oblate states which were also found in [30, 31].

The color in Figure 4 indicates the value of apparent surface charge $Q=[\varepsilon \mathbf{E} \cdot \mathbf{n}]=-\left[\phi_{n}\right]$. In the beginning $(T=0)$, a non-uniform surface charge density is observed due to the imbalanced charging rates of the vesicle and the surrounding fluid. At this stage, the resultant electric compressive force is vertical so the vesicle tends to become an oblate shape. After the vesicle membrane is charged, the resultant compressive force becomes horizontal thus the vesicle turns to be the prolate shape again. From the transmembrane potential Eq. (32), one can also see that as an equilibrium state, the surface charge density $Q$ should approach to zero as time progresses. This is exactly what we see from Figure 4.
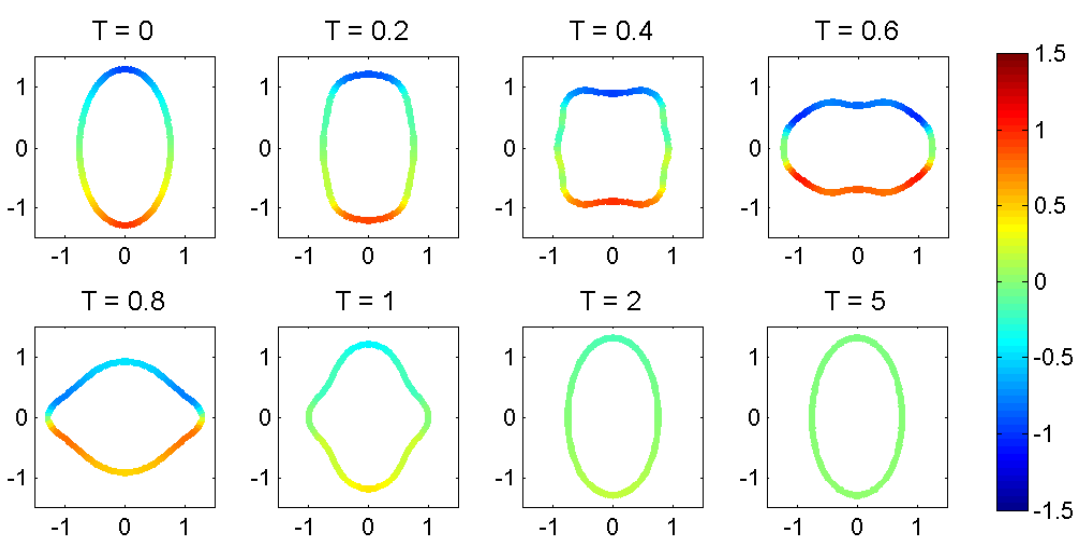

Figure 4: Snapshots for the POP transition with capillary number $C a=10$. The color coding indicates the magnitude of the surface charge density $Q$.

While increasing the capillary number to $C a=10^{3}$ (which corresponds to the typical vesicle bending modulus $c_{b}=10^{-19} \mathrm{~J}$ ), as shown in Figure 5, we surprisingly find that the "wrinkling" instability occurs on the vesicle membrane during the POP transition. We attribute such shape instability to the intensity of applied electric field. As in [39], the authors have indicated that above a critical electric field strength $E_{c}=2 V_{c} / 3 R$, vesicles may porate in a finite critical time $t_{c}=-t_{m m} \ln \left(1-2 V_{c} / 3 R E_{c}\right)$. Therefore, in their experiments, they have developed a two-step electric pulse technique (a stronger pulse above $E_{c}$ is applied in a very short period $\left(T \ll t_{c}\right)$ while a much weaker pulse is applied afterwards) to avoid the vesicle poration so that the POP transition can be observed. Since the critical poration voltage $V_{c}$ for lipid membranes is around $1 \mathrm{~V}$ [4], the critical Mason number is around $M n_{c}=$ 3.5 in the present setting. Here, the present Mason number $M n=20$ is above the critical value for electroporation, so one can expect that vesicle poration should occur. However, the current immersed boundary method with Lagrangian type of interface representation cannot handle this topological change of vesicle shape exactly so the oscillatory wrinkling phenomenon appears instead in Figure 5. One should also notice that the occurrence of wrinkling instability is not just for our present numerical method, we have implemented the code of boundary integral method (similar to the one developed in [31]) and run the 

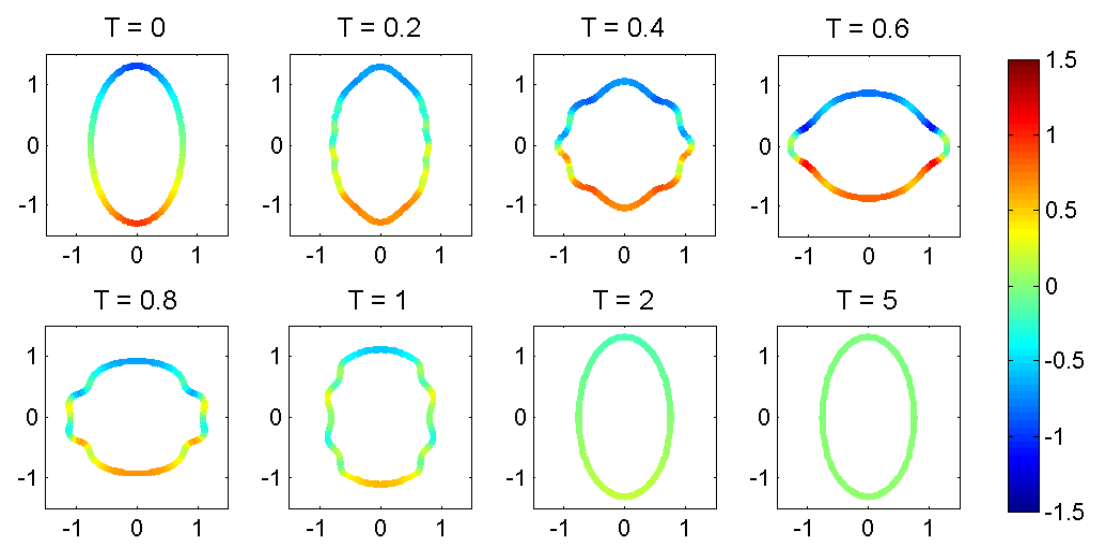

Figure 5: Snapshots for the POP transition with capillary number $C a=10^{3}$, the wrinkling instability is observed on the vesicle membrane.

simulation with the same chosen parameters as in Figure 5. Surprisingly, we have obtained similar wrinkling phenomenon as shown in Figure 5. This wrinkling instability has also been found by imposing a vesicle in a strong elongation flow in experiment [20] and in two-dimensional simulation [27]. As the results shown in Figure 4 and 5, our numerical simulations suggest that the vesicle with larger bending rigidity can prevent the wrinkling instability and its dynamics from electroporation.

\subsection{Effect of membrane conductance $G$}

Next we investigate the effect of membrane conductance on the POP transition. To quantify the vesicle shape, we define the deformation number $D$ by $D=\frac{L}{B}$, where $L$ and $B$ are the corresponding vertical and horizontal lengths of the vesicle. So the deformation number $D>1$ is prolate shape while $D<1$ is oblate shape.

We study the effect of membrane conductance by varying the conductance: $G=$ $0,0.05,0.2$. Note that, the nonzero membrane conductance means the lipid bilayer membrane contains ion channels or pores and charges may transport across the membrane. The time evolution of deformation number $D$ is given in Figure 6. It is interesting to see that, in the case of $G=0.05$, the vesicle still undergoes a POP transition but with slightly longer charging time compared to the case of $G=0$. However, by increasing the conductance to $G=0.2$, the vesicle turns to an oblate shape and POP does not occur. We attribute this peculiar behavior to a weak restoring electric force at this stage so the bending force is too large to overcome. Such behavior has also been found in the work [30]. Figure 7 shows the snapshots of the vesicle shape and its surface charge density $Q$. Different from the previous case of the non-conducting membrane $G=0$ in Figure 4, we find polarized charge distribution on the vesicle due to the leakage of ions for $G=0.2$. 


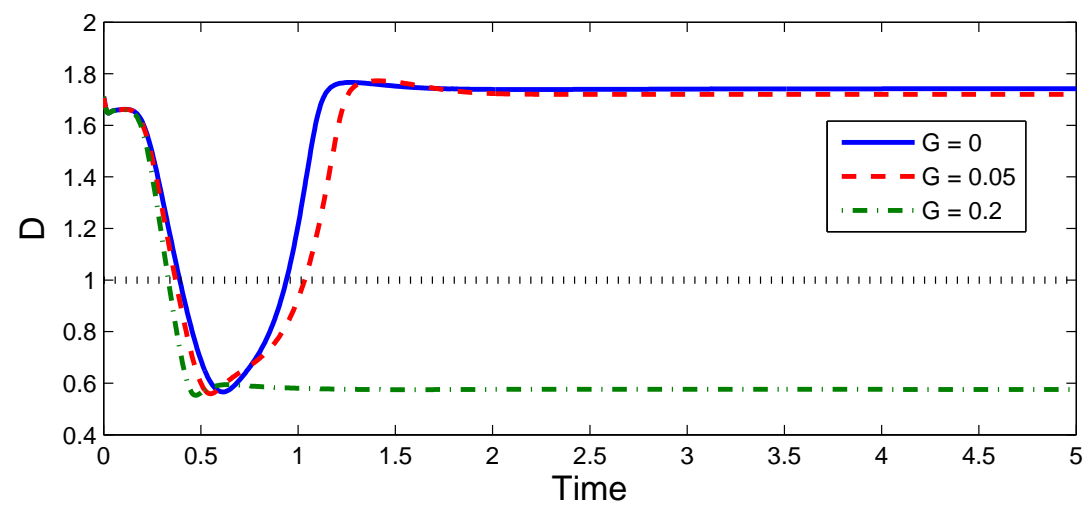

Figure 6: The time evolution of deformation number $D=L / B$ for $G=0,0.05,0.15$ with $C=0.1$.
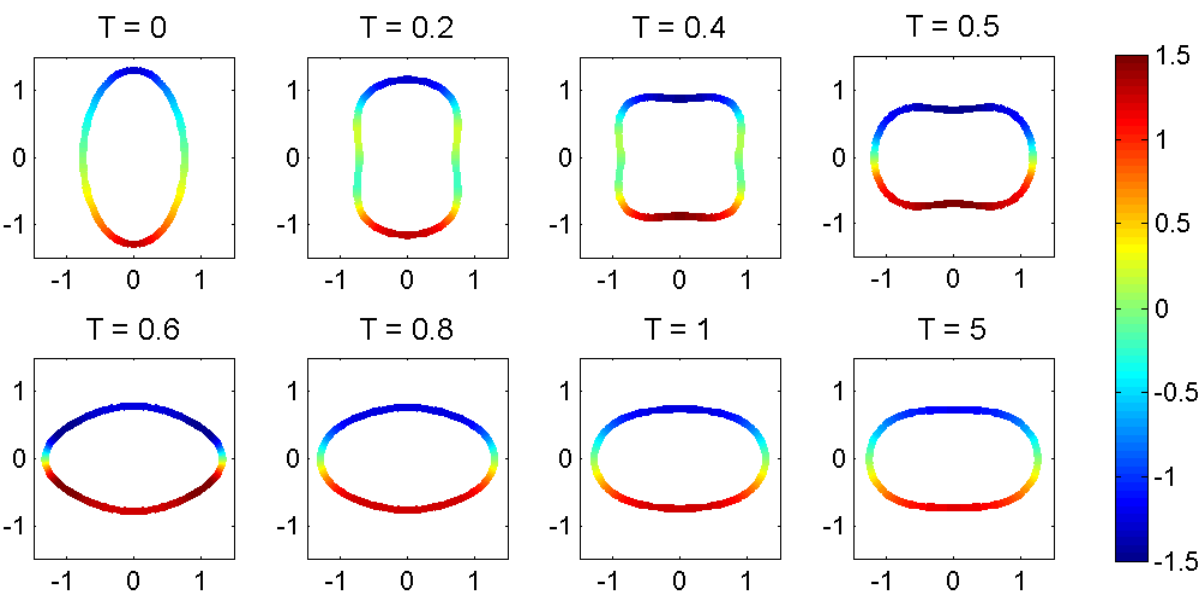

Figure 7: Snapshots of vesicle electrodeformation with $G=0.2$. The color coding indicates the magnitude of surface charge density $Q$.

\subsection{Effect of Reynolds number}

In this test, we investigate the effect of Reynolds number on the vesicle electrodeformation. We set the membrane conductance $G=0$, and vary the Reynolds number from the Stokes regime to the Navier-Stokes regime: $R e=0.02,0.2,2$. The results are shown in Figure 8. For $R e=0.02$ and 0.2 , the vesicle experiences POP transition, and the charging time of the case $R e=0.2$ is slightly larger than to the one of $R e=0.02$. However, as the Reynolds number increases to $R e=2$, the POP transition does not occur despite the electric stress still tends to elongate the vesicle into an oblate shape in a very short time. We attribute such damping on the POP transition to the effect of inertia, which results in a fast convection of surface charge. Nevertheless, all three cases converge to the same equilibrium prolate shape. 


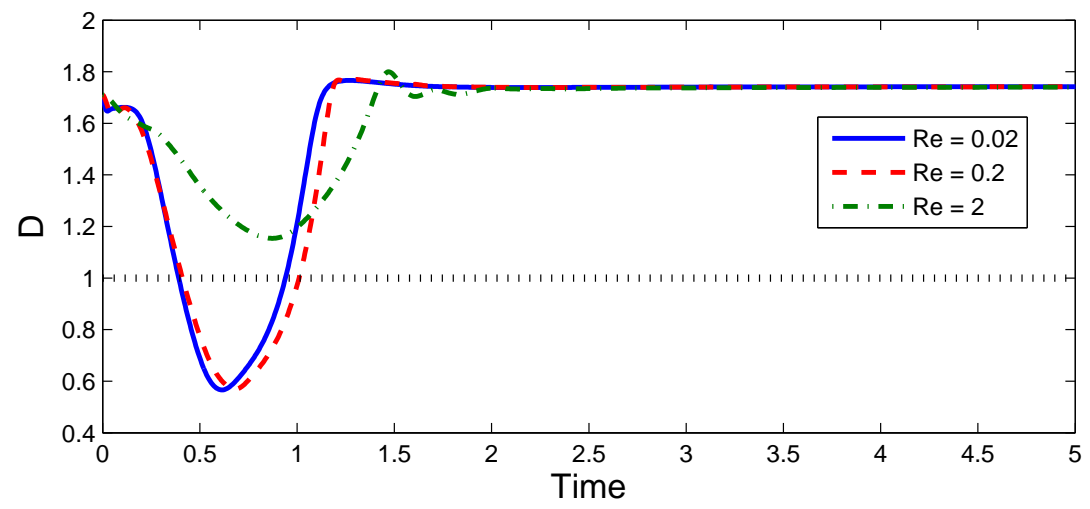

Figure 8: The time evolution of deformation number $D$ for $R e=0.01,0.1,1$ with fixed $C=0.1$ and $G=0$.

\subsection{Combination of shear flow and electric field}

Under a planar shear flow a vesicle with small viscosity contrast $\left(\mu_{r}=\mu^{-} / \mu^{+}\right)$may exhibit a tank-treading motion on the membrane as the vesicle shape remains stationary with a tangential tank-treading motion along the membrane. However, above the critical viscosity contrast, the vesicle motion transitions to a tumbling motion when the vesicle undergoes a rigid body rotation in shear flow. To further validate our numerical algorithms, we study the combined effect of the vesicle dynamics under shear flow and electric field simultaneously as in previous work $[22,30]$. More precisely, our main focus in these simulations is to investigate how the electric field affects the vesicle behavior in a shear flow.

To properly take into account the unmatched viscosity of the interior and bulk fluids, the viscous term $\frac{1}{R e} \Delta \mathbf{u}$ in the momentum Eq. (26) is replaced by $\frac{1}{R e} \nabla \cdot\left(\mu(\mathbf{x})\left(\nabla \mathbf{u}+\nabla \mathbf{u}^{T}\right)\right)$, where $\mu(\mathbf{x})$ is a piecewise constant $\left(\mu=\mu^{-}\right.$in $\Omega^{-}$and $\mu=\mu^{+}$in $\left.\Omega^{+}\right)$in the computational domain $\Omega$. (Note that, the characteristic viscosity in the non-dimensionalization in Section 4 is replaced by $\mu^{+}$.) We leave the details of numerical discretization for the momentum equation with unmatched viscosity in Appendix. In the following tests, we simulate the vesicle electrodeformation under a shear flow with shear rate $\chi=0.8$ and an electric field. Again we set the membrane conductance $G=0$.

Matched viscosity

In this test we study the case of matched viscosity $\mu_{r}=1$. The tank-treading motion is quantified by measuring the inclination angle $\theta$ between the long axis of the vesicle and the flow direction. We run the simulations with different Mason number $M n=0,1,20$, a measure for the strength of applied electric field (no electric field applied for $M n=0$ ). Figure 9(a) shows the time evolution of the inclination angle. For $M n=1$ the vesicle undergoes tank-treading motion as the $M n=0$ case. However, the inclination angle for $M n=1$ case is larger than the one for $M n=0$. This is expected as the electric field is applied in the cross-stream direction. For the strongest electric field $M n=20$ case, the vesicle first attempts the tumbling motion then it reaches a tank-treading motion as in the cases of $M n=0,1$. The snapshots of the case $M n=20$ are shown in Figure 9(b). 
These findings show that the equilibrium inclination angle increases as the Mason number $M n$ increases (i.e., the intensity of electric field increases). This is because the electric force acting on the vesicle membrane tends to align the vesicle and elongate its shape along the electric field direction. The larger electric field strength $(M n)$, the larger inclination angle will be. Similar results and conclusions have also been obtained in $[22,30]$.

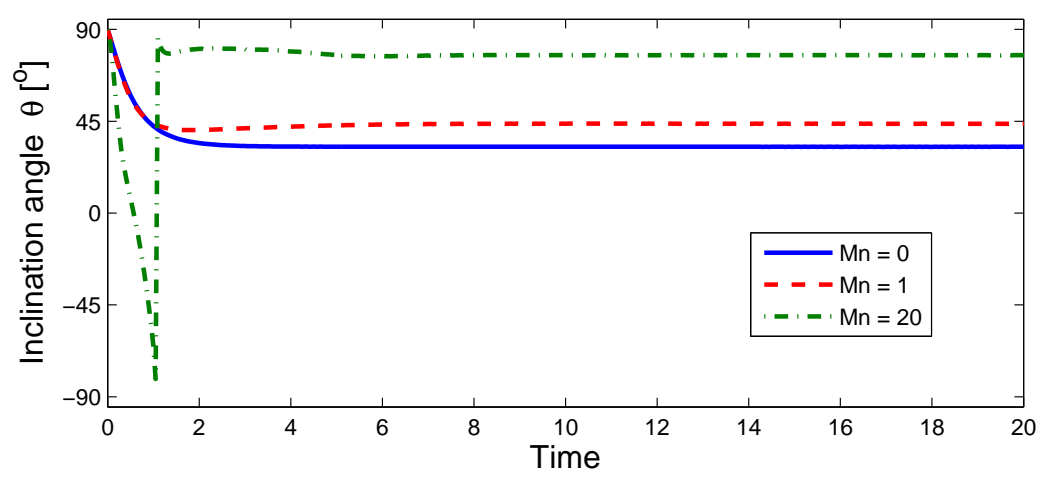

(a)
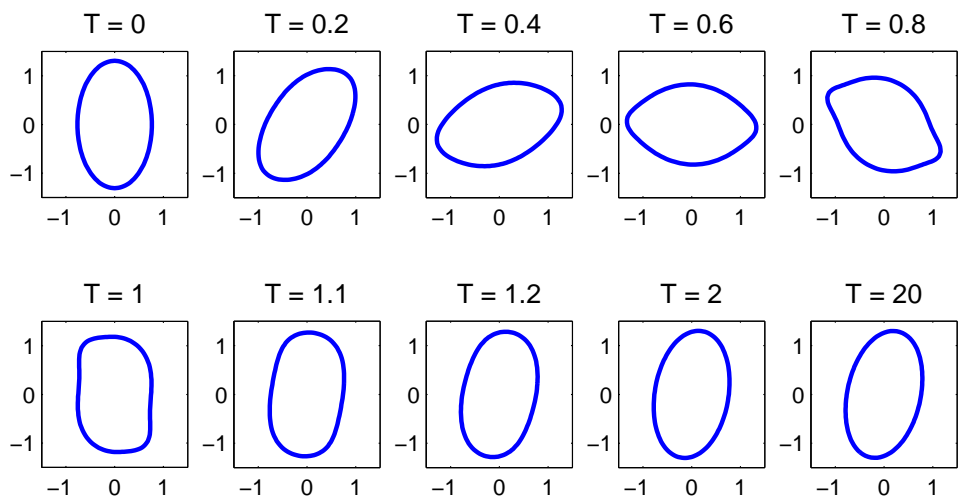

(b)

Figure 9: (a) The inclination angles for $M n=0,1,20$. (b) Snapshots of the vesicle electrohydrodynamics under a shear flow with $M n=20$.

\section{Unmatched viscosity}

In this test, we set the viscosity contrast $\mu_{r}=20$ to make sure the vesicle tumbles under shear flow in the absence of an electric field. Because the inertia effect might inhibit the tumbling motion [19, 24], here we set the Reynolds number $R e=0.02$ to ensure the flow is in the Stokes regime. As in the matched viscosity case, we run the simulations with different Mason number $M n=0,1,20$ to see how the electric field affects the tumbling motion.

Figure 10(a) shows the time evolution of the inclination angle. For the weak electric field case $M n=1$, the vesicle undergoes tumbling motion as the one without the electric field $M n=0$. However, the tumbling period is longer in the $M n=1$ case than the $M n=0$ case. For the stronger electric field case $M n=20$, the tumbling motion ceases and the 
vesicle undergoes tank-treading instead. Figure 10(b) shows the snapshots of the vesicle dynamics for the cases of $M n=0$ and $M n=20$. In these simulations, we have observed that by increasing the intensity of electric field, the vesicle tumbling motion under shear flow is damped out by the electric field in the cross-stream direction. This finding is in good agreement with those in asymptotic theory [37] and numerical simulations [22, 30].

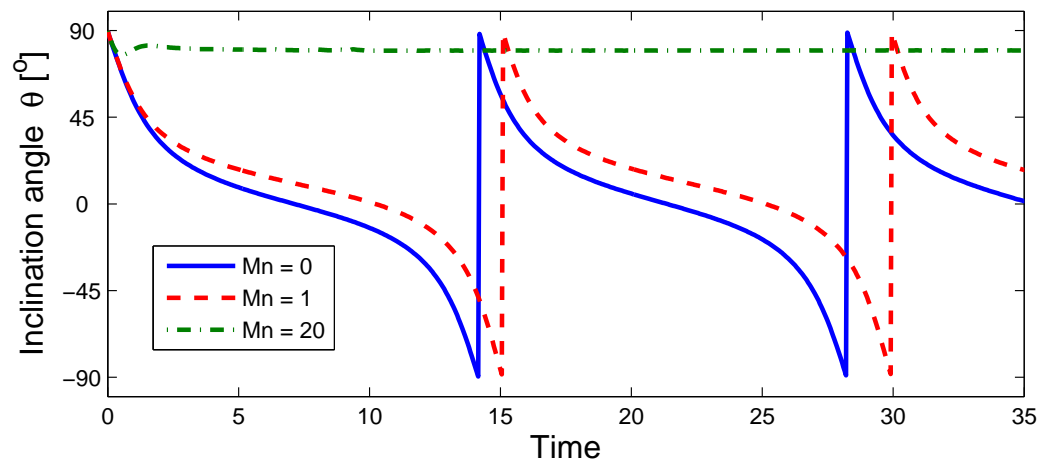

(a)
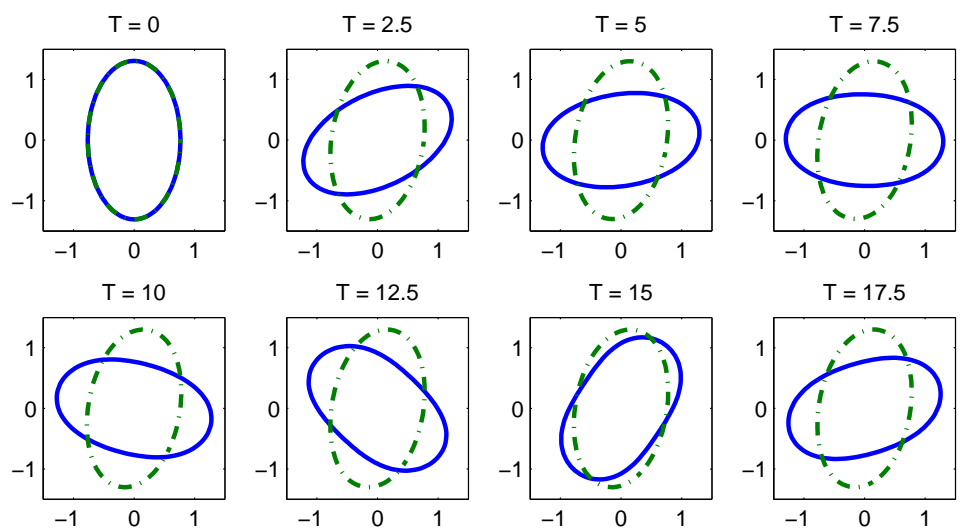

(b)

Figure 10: (a) The inclination angles for $M n=0,1,20$. (b) Snapshots of vesicle electrohydrodynamics under a shear flow. Solid line '-': $M n=0$; dashed-dotted line '-.-': $M n=20$.

\section{Conclusions}

In this paper, we have developed a coupled immersed boundary (IB) and immersed interface method (IIM) to simulate vesicle electrohydrodynamics in Navier-Stokes leaky dielectric fluids. The electric potential and transmembrane potential are numerically solved by the immersed interface method with the jump condition along the normal direction. Instead of applying an electric volume force to the fluid system, we treat the electric effect as an interfacial force with a normal jump due to the Maxwell stress on the interface. Therefore, 
the vesicle electrohydrodynamics can be cast in a unified immersed boundary formulation, which greatly simplifies the numerical scheme for the leaky dielectric electrohydrodynamic simulations.

To carefully validate our numerical algorithms, we first check the accuracy of the present immersed interface method for solving the electric potential. We further perform the convergence study for the fluid variables. We then conduct a series of simulations to investigate the dynamics of prolate-oblate-prolate transition of a vesicle. We focus on studying the effect of membrane conductance and Reynolds number on the POP transition. Furthermore, we investigate how the electric field affects the vesicle dynamics in a shear flow. In the future, we shall extend our present methodology to the case with AC electric field. We also plan to extend the present $2 \mathrm{D}$ scheme to $3 \mathrm{D}$ axisymmetric case or even fully $3 \mathrm{D}$ simulations.

\section{Acknowledgments}

M.-C. Lai acknowledges support by Ministry of Science and Technology of Taiwan under research grant MOST-104-2115-M-009-014-MY3 and NCTS. Y.-N. Young acknowledges support from NSF-DMS 1009105 and 1222550.

\section{Appendix}

In the appendix, we introduce a simple numerical discretization to solve the momentum equation in Navier-Stokes flow with variable viscosity. The momentum equation with variable viscosity is

$$
\frac{\partial \mathbf{u}}{\partial t}+(\mathbf{u} \cdot \nabla) \mathbf{u}=-\nabla p+\frac{1}{R e} \nabla \cdot(2 \mu(\mathbf{x}) D)+\mathbf{f},
$$

where $\mu(\mathbf{x})$ is a piecewise constant and $D=\frac{1}{2}\left(\nabla \mathbf{u}+\nabla \mathbf{u}^{T}\right)$ is the deformation tensor. In practice, the viscosity can be calculated through the harmonic averaging as

$$
\frac{1}{\mu(\mathbf{x})}=\frac{H(\mathbf{x})}{\mu^{-}}+\frac{1-H(\mathbf{x})}{\mu^{+}}
$$

where $H(\mathbf{x})$ is the indicator function (or regularized Heaviside function) that can be obtained by solving the Poisson equation

$$
\Delta H(\mathbf{x})=-\nabla \cdot \int_{\Sigma} \mathbf{n}(s) \delta(\mathbf{x}-\mathbf{X}(s))\left|\mathbf{X}_{s}\right| \mathrm{d} s .
$$

In order to fully exploit Fast Fourier Transform (FFT) in our immersed boundary solver, we adopt the idea used in [5] and split the variable viscosity term into two parts: a constant coefficient part is treated implicitly, and a variable coefficient part is treated explicitly. For instance, the first-order backward Euler time-stepping scheme for Eq. (34) in the projection method has the form as

$$
\frac{\mathbf{u}^{*}-\mathbf{u}}{\Delta t}+\left(\mathbf{u}^{n} \cdot \nabla_{h}\right) \mathbf{u}^{n}=-\nabla_{h} p^{n}+\frac{1}{R e}\left(\lambda \Delta_{h} \mathbf{u}^{*}-\lambda \Delta_{h} \mathbf{u}^{n}+\nabla_{h} \cdot(2 \mu D)^{n}\right)+\mathbf{f}^{n},
$$

where $\lambda=\max \left(\mu^{-}, \mu^{+}\right)$. One can immediately see that the above discretization involves solving a constant coefficient elliptic equation for the intermediate velocity $\mathbf{u}^{*}$ which can be solved efficiently by using FFT or the fast direct solver provided by Fishpack [1]. 


\section{References}

[1] J. Adams, P. Swarztrauber, R. Sweet, Fishpack - a package of Fortran subprograms for the solution of separable elliptic partial differential equations, 1980. Available at http://www.netlib.org/fishpack

[2] S. Aranda, K. A. Riske, R. Lipowsky, and R. Dimova, Morphological transitions of vesicles induced by alternating electric fields, Biophysical J., 108 (2008), L19-L21.

[3] R. Dimova, K. A. Riske, S. Aranda, N. Bezlyepkina, R. L. Knorr, and R. Lipowsky, Giant vesicles in electric fields, Soft Matter, 3 (2007), 817-927.

[4] R. Dimova, N. Bezlyepkina, M. D. Jordo, R. L. Knorr, K. A. Riske, M. Staykova, P. M. Vlahovska, T. Yamamoto, P. Yang, and R. Lipowsky, Vesicles in electric fields: Some novel aspects of membrane behavior, Soft Matter, 5 (2009), 3201-3212.

[5] T. G. Fai, B. E. Griffith, Y. Mori, C. S. Peskin, Immersed boundary method for variable viscosity and variable density problems using fast constant-coefficient linear solvers I: Numerical method and results, SIAM J. Sci. Comput., 35 (2013), B1132-B1161.

[6] C. Grosse and H. P. Schwan, Cellular membrane potentials induced by alternating fields, Biophys J., 63 (1992), 1632-642.

[7] B.E. Griffith, On the volume conservation of the immersed boundary method, Commun. Comput. Phys., 12 (2012), 401-432.

[8] F. H. Harlow, J. E. Welsh, Numerical calculation of time-dependent viscous incompressible flow of fluid with a free surface, Phys. Fluids, 8 (1965), 2181-2189.

[9] W. Helfrich, Elastic properties of lipid bilayers - theory and possible experiments. Z. Nat., 28c (1973), 679-703.

[10] H. Hyuga, K. Kinosita, Jr., and N. Wakabayashi, Deformation of vesicles under the influence of strong electric fields, Jpn. J. Appl. Phys., 30 (1991), 1141-1148.

[11] H. Hyuga, K. Kinosita, Jr., and N. Wakabayashi, Deformation of vesicles under the influence of strong electric fields II, Jpn. J. Appl. Phys., 30 (1991), 1333-1335.

[12] H. Hyuga, K. Kinosita, Jr., and N. Wakabayashi, Transient and steady-state deformations of a vesicle with an insulating membrane in response to step-function or alternating electric fields, Jpn. J. Appl. Phys., 30 (1991), 2649-2656.

[13] C. K. Haluska, K. A. Riske, V. Marchi-Artzner, J.-M. Lehn, R. Lipowsky, and R. Dimova, Time scales of membrane fusion revealed by direct imaging of vesicle fusion with high temporal resolution, PNAS, 103 (2006), 15841-15846.

[14] W.-F. Hu, Y. Kim, M.-C. Lai, An immersed boundary method for simulating the dynamics of three-dimensional axisymmetric vesicles in Navier-Stokes flows, J. Comput. Phys., 257 (2014), 670-686. 
[15] W.-F. Hu, M.-C. Lai, and Y.-N. Young, A hybrid immersed boundary and immersed interface method for electro-hydrodynamic simulations, J. Comput. Phys., 282 (2015), 47-61.

[16] K. Kinosita, Jr., I. Ashikawa, N. Saita, H. Yoshimura, H. Itoh, K. Nagayama, and A. Ikegami, Electroporation of cell membrane visualized under a pulsed-laser fluorescence microscope, Biophys J., 53 (1988), 1015-1019.

[17] B. Kaoui, G. H. Ristow, I. Cantat, C. Misbah, and W. Zimmermann, Lateral migration of a two-dimensional vesicle in unbounded Poiseuille flow, Phys. Rev. E, 77 (2008), 021903 .

[18] R. L. Knorr, M. Staykova, R. S. Gracia and R. Dimova, Wrinkling and electroporation of giant vesicles in the gel phase, Soft Matter, 6 (2010), 1990-1996.

[19] Y. Kim, M.-C. Lai, Numerical study of viscosity and inertial effects on tank-treading and tumbling motions of vesicles under shear flow, Phys. Rev. E, 86 (2012), 066321.

[20] V. Kantsler, E. Segre, and V. Steinberg, Vesicle dynamics in time-dependent elongation flow: Wrinkling instability, Phys. Rev. Lett., 99 (2007), 178102.

[21] E. M. Kolahdouz, D. Salac, A numerical model for the trans-membrane voltage of vesicles, Applied Mathematics Letters, 39 (2015), 7-12.

[22] E. M. Kolahdouz, D. Salac, Electrohydrodynamics of three-dimensional vesicles: A numerical approach, SIAM J. Sci. Comput., 37 (2015), B473-B494.

[23] E. M. Kolahdouz, D. Salac, Dynamics of three-dimensional vesicles in DC electric fields, Phys. Rev. E, 92 (2015), 012302.

[24] A. Laadhari, P. Saramito, C. Misbah, Vesicle tumbling inhibited by inertia, Phys. Fluids, 24 (2012), 031901.

[25] M.-C. Lai, H.-C. Tseng, A simple implementation of the immersed interface methods for Stokes flows with singular forces, Computers and Fluids, 37 (2008), 99-106.

[26] Z. Li, K. Ito, The Immersed Interface Method, SIAM 2006.

[27] K. Liu, S. Li, Nonlinear simulations of vesicle wrinkling, Math. Meth. Appl. Sci., 37 (2014), 1093-1112.

[28] C. B. Macdonald, S. J. Ruuth, The implicit closest point method for the numerical solution of partial differential equations on surfaces, SIAM J. Sci. Comput., 31(6) (2009), 4330-4350.

[29] J. R. Melcher, G. I. Taylor, Electrohydrodynamics: A review of the role of interfacial shear stresses, Annu. Rev. Fluid Mech., 1 (1969), 111.

[30] L. C. McConnell, M. Miksis, P. M. Vlahovska, Vesicle electrohydrodynamics in DC electric fields, IMA J. Appl. Math., (2013), 1-21. 
[31] L. C. McConnell, P. M. Vlahovska and M. J. Miksis, Vesicle dynamics in uniform electric fields: squaring and breathing, Soft Matter, 11 (2015), 4840-4846.

[32] H. Nganguia and Y.-N. Young, Equilibrium electrodeformation of a spheroidal vesicle in an ac electric field, Phys. Rev. E, 88 (2013), 052718.

[33] D. Russel, Z. J. Wang, A Cartesian grid method for modeling multiple moving objects in 2D incompressible viscous flow, J. Comput. Phys., 191 (2003), 177-205.

[34] K. A. Riske and R. Dimova, Electro-deformation and poration of giant vesicles viewed with high temporal resolution, Biophysical J., 88 (2005), 1143-1155.

[35] M. M. Sadik, J. Li, J. W. Shan, D. I. Shreiber and H. Lin, Vesicle deformation and poration under strong de electric fields, Phys. Rev. E, 83 (2011), 066316

[36] D. A. Saville, Electrohydrodynamics: The Taylor-Melcher leaky dielectric model, Annu. Rev. Fluid Mech., 29 (1997), 27.

[37] J. T. Schwalbe, P. M. Vlahovska, and M. J. Miksis, Vesicle electrohydrodynamics, Phys. Rev. E, 83 (2011), 046309.

[38] J. Seiwert, M. J. Miksis and P. M. Vlahovska, Stability of biomimetic membranes in DC electric fields, J. Fluid. Mech., 706 (2012), 58-70.

[39] P. F. Salipante and P. M. Vlahovska, Vesicle deformation in DC electric pulses, Soft Matter, 10 (2014), 3386-3393.

[40] Y. Seol, W.-F. Hu, Y. Kim, M.-C. Lai, An immersed boundary method for simulating vesicle dynamics in three dimensions, submitted for publication.

[41] G.I. Taylor, Studies in electrohydrodynamics I. The circulation produced in a drop by an electric field, Proc. R. Soc. London A, 291 (1966), 159.

[42] L. N. Trefethen, Spectral Methods in MATLAB, SIAM, Philadelphia, 2000

[43] P. M. Vlahovska, R. S. Gracia, S. Aranda-Espinoza, and R. Dimova, Electrohydrodynamic model of vesicle deformation in alternating electric field, Biophys J., 96 (2009), 4789-4803.

[44] S. Xu, An iterative two-fluid pressure solver based on the immersed interface method, Commun. Comput. Phys., 12 (2012), 528-543.

[45] X. Yang, X. Zhang, Z. Li, G.-W. He, A smoothing technique for discrete delta functions with application to immersed boundary method in moving boundary simulations, J. Comput. Phys., 228 (2009) 7821-7836.

[46] J. Zhang, J. D. Zahn, W. Tan, and H. Lin, A transient solution for vesicle electrodeformation and relaxation, Phys. Fluids, 25 (2013), 071903.

[47] J. Zhang, J. D. Zahn and H. Lin, Transient solution for droplet deformation under electric fields, Phys. Rev. E, 87 (2013), 043008. 